\section{Mortalidade de idosos com desnutrição em Belo Horizonte, Minas Gerais, Brasil: uma análise multidimensional sob o enfoque de causas múltiplas de morte}

\author{
Mortality of malnourished elderly in Belo Horizonte, \\ Minas Gerais State, Brazil: a multidimensional \\ analysis focusing on multiple causes of death
}

\author{
1 Escola de Enfermagem, \\ Universidade Federal de \\ Minas Gerais, Belo Horizonte, \\ Brasil. \\ 2 Escola de Veterinária, \\ Universidade Federal de \\ Minas Gerais, Belo Horizonte, \\ Brasil. \\ 3 Secretaria Municipal de \\ Saúde de Belo Horizonte, \\ Belo Horizonte, Brasil. \\ Correspondência \\ E. M. Rezende \\ Departamento de \\ Enfermagem Materno- \\ Infantil e Saúde Pública, \\ Escola de Enfermagem, \\ Universidade Federal de \\ Minas Gerais. \\ Av. Alfredo Balena 190 \\ Belo Horizonte, $M G$ \\ 30130-100, Brasil. \\ ednarez@enf.ufmg.br
}

\begin{abstract}
This study aimed to identify the mortality profiles of elderly individuals with malnutrition, based on associations between multiple causes of death and other variables recorded on the death certificate. We studied the deaths of elderly $(\geq 60$ years $)$ living in Belo Horizonte, Minas Gerais State, Brazil, with malnutrition recorded as one of the causes. Data were used from the mortality information system. Multiple correspondence analysis was used to investigate these associations. The study showed excess mortality among women, whites, widowers, and individuals with low schooling. Ten mortality profiles were studied, and malnutrition was five times more frequent according to the multiple-causes technique. The results showed both the importance of multiple causes of death not detected by focusing on the underlying cause and the adequacy of multiple correspondence analysis for identifying the associations between causes and other variables listed on the death certificate.
\end{abstract}

Aged; Malnutrition; Multiple Cause of Death
Edna Maria Rezende 1

Ivan Barbosa Machado Sampaio 2

Lenice Harumi Ishitani 3

Eunice Francisca Martins 1

Lenice de Castro Mendes Vilella ${ }^{1}$

Até os anos 60 o Brasil era uma país extremamente jovem, com cerca de $52 \%$ de sua população abaixo de 20 anos e menos de $3 \%$ acima de 65 anos. Ao final da década, com a mortalidade em queda e o rápido declínio da fecundidade, iniciou-se um processo de desestabilização da estrutura etária da população brasileira 1. Observou-se, a partir de então, um crescimento relativo da proporção de idosos, configurando um aumento de $500 \%$ entre 1960 e 2002 2. Atualmente, representam $9,2 \%$ da população total, ultrapassado 15 milhões de brasileiros 3 . Para 2025 há uma projeção de aproximadamente 32 milhões de pessoas idosas 4 .

Este aumento progressivo de idosos coloca em discussão grandes desafios relacionados ao envelhecimento, entre eles as novas demandas para os serviços de saúde e os custos adicionais com assistência médica e social 2 . Além disso, o momento de profunda desordem econômica em que essa transição vem ocorrendo deixa a população idosa, especialmente a de baixa renda, mais desamparada e carente, configurando um quadro crítico de exclusão social do idoso 5 . Paralelamente às mudanças na composição etária populacional, modificações vêm ocorrendo também na incidência e prevalência das doenças, bem como nas principais causas de morte. Doenças crônico-degenerativas de longa dura- 
ção, transtornos mentais e afecções de etiologia cada vez mais complexas vão se acumulando na população idosa, considerando o aumento progressivo da expectativa de vida.

Entre os problemas clínicos mais usuais em idosos, os distúrbios nutricionais têm merecido papel de destaque, como é o caso da desnutrição protéico-calórica, umproblemamultinutricional, secundário à deficiência de um ou mais nutrientes essenciais 6 e caracterizada por distúrbios clínicos causados por vários graus de deficiência 2 . O termo desnutrição protéico-calórica engloba dois tipos 7: marasmo (má nutrição quantitativa, hipoalimentação global ou calórica, subnutrição), que se caracteriza pela falta de calorias e proteínas; Kwashiokor (má nutrição qualitativa, predominantemente protéica), em que ocorrem distúrbios metabólicos diversos em estados mais graves. Além do número excessivo de óbitos que vem causando a cada ano, a desnutrição protéico-calórica agrava as doenças em atividade, torna maior a susceptibilidade a infecções e aumenta os riscos para outras morbidades, reduzindo consideravelmente a qualidade de vida 8,9 .

O risco de morrer no Brasil por desnutrição, durante a velhice, apresenta tendência crescente, relacionado a problemas sociais e desigualdades no acesso a alimentação adequada 10 . A má nutrição no idoso pode estar associada ainda a alterações fisiológicas do envelhecimento, a condições sócio-econômicas, a doenças que diminuem o apetite e absorção dos alimentos e a interação com medicamentos, já que os fármacos podem afetar a ingestão e o metabolismo de nutrientes. Figuram também como causas da desnutrição no idoso o desconhecimento sobre o preparo dos alimentos e a nutrição adequada, problemas dentários, limitações físicas, dificuldade de visão e deambulação, tremores, isolamento social, depressão, problemas mentais e alcoolismo 11,12.

Embora associada ao aumento do número de óbitos, a desnutrição não tem sido devidamente evidenciada nas estatísticas oficiais de mortalidade. Isto porque, ao se tabular apenas uma causa - a causa básica de morte -, há uma perda de grande parte das informações 13,14. Otero et al. 15, ao analisarem a mortalidade por desnutrição em idosos sob o enfoque de causa básica, para as regiões metropolitanas dos estados do Rio de Janeiro e São Paulo, recomendaram a realização de estudos considerando as causas múltiplas de morte. Em Belo Horizonte, Minas Gerais, a exemplo de outras capitais da Região Sudeste, embora a proporção de idosos venha crescendo a cada ano, nenhum estudo de mortes por desnutrição, sob esse enfoque específico, foi ainda realizado. Rezende 16 , ao analisar de modo geral as causas múltiplas de óbitos em Belo Horizonte, detectou que a desnutrição ocorre cerca de cinco vezes mais do que os índices divulgados pelas estatísticas oficiais de mortalidade e que mais de $60 \%$ dos casos ocorrem em pessoas com mais de 60 anos.

O estudo da associação de causas múltiplas de morte, por levar em consideração todas as causas mencionadas na Declaração de Óbito, poderá expressar a real magnitude da desnutrição como causa básica ou associada de morte em idosos. Procurou-se, no presente estudo, identificar perfis de mortalidade de idosos desnutridos com base nas associações entre causas de morte e outras variáveis da Declaração de Óbito, utilizando a análise multidimensional sob o enfoque de causas múltiplas de morte.

\section{Método}

Foram estudadas as mortes ocorridas no período de 2000 a 2003, de pessoas com 60 anos e mais, residentes em Belo Horizonte, que tinham a desnutrição como uma das causas mencionadas na Declaração de Óbito, independente de sua posição, representada nas categorias de E40 a E46 da 10a revisão da Classificação Internacional de Doenças (CID-10) 17. Assim, foi analisada não apenas quando selecionada como causa básica da morte, mas também sob o enfoque de causas múltiplas 13. Utilizou-se o banco de dados do Sistema de Informações sobre Mortalidade (SIM), disponibilizado em CD-ROM pela Secretaria Municipal de Saúde de Belo Horizonte.

A codificação das causas de morte foi feita com base na CID-10 17, as quais foram agrupadas de acordo com as Listas Especiais de Tabulação de Mortalidade e também com base nas freqüências iniciais das menções. Para as causas agrupadas em um nível de agregação maior do que aquele em que foram mencionadas na Declaração de Óbito, garantiu-se que todas as duplicações e multiplicações de diagnósticos fossem eliminadas, conforme sugerido por Santo 13. A desnutrição como causa de morte foi analisada de forma desagregada em três categorias: desnutrição protéica $(\mathrm{KWH})$ - E40; marasmo nutricional (MRT) - E41; desnutrição protéico-calórica (DPC) - E43 a E46. A categoria E42 (Kwashiorkor marasmático) não foi avaliada por não haver menção a esta categoria nas Declarações de Óbitos estudadas. Para cada grupo das demais causas estudadas, duas categorias foram criadas indicando a presença da enfermidade (S) ou a sua ausência (N). Assim, a variável causa ficou denominada pela letra e número do grupo correspondente seguidos de "S" ou "N". Procurou-se, sempre que possível, manter para cada grupo de causa a letra correspondente ao agrupamento já estabelecido 
na CID-10: doenças respiratórias (J), doenças circulatórias (I), doenças neurológicas (G) etc. Pneumonia, por exemplo, ficou representada por J2; pneumonia de aspiração, por J4; doenças cerebrovasculares, por I7; aterosclerose, por I8. Quando presente, a pneumonia foi expressa por J2S; quando ausente, por J2N. De forma similar procedeu-se para os demais grupos.

Além das causas de morte, outras variáveis estudadas na Declaração de Óbito foram sexo, idade, escolaridade, mês de ocorrência, raça, estado civil, local de ocorrência do óbito, distrito de residência e assistência médica, categorizadas como mostra a Tabela 1. Para a variável sexo, foram estabelecidas as categorias masculino (MAS) e feminino (FEM). A idade foi estratificada em quatro categorias: ID1 (60-69 anos), ID2 (70-79 anos), ID3 (80-89 anos) e ID4 (90 anos e mais). Seis categorias foram criadas para a escolaridade: ES1 (1-3 anos de estudo), ES2 (4-7 anos de estudo), ES3 (8-11 anos de estudo), ES4 (12 anos e mais de estudo), ESG (escolaridade ignorada) e NEH (nenhuma escolaridade). A variação sazonal de ocorrência do óbito foi avaliada com base no mês de ocorrência. Óbitos ocorridos no período de calor (CAL) foram aqueles que aconteceram nos meses de outubro a abril; ocorridos no frio (FRI) foram os registrados nos meses de maio a setembro. Os meses frios foram os que apresentaram temperatura média compensada menor ou igual a $21,3^{\circ} \mathrm{C}$ e temperatura mínima absoluta menor ou igual a $13,3^{\circ} \mathrm{C}$. Meses considerados quentes foram os que registraram temperaturas acima dos valores mencionados para

Tabela 1

Categoria, freqüência e percentual das variáveis das Declarações de Óbitos de idosos com desnutrição. Belo Horizonte, Minas Gerais, Brasil, 2000 a 2003.

\begin{tabular}{|c|c|c|}
\hline Variáveis/Categorias & Freqüência & $\%$ \\
\hline \multicolumn{3}{|l|}{ Idade (anos) } \\
\hline ID1 (60-69) & 303 & 21,5 \\
\hline ID2 (70-79) & 382 & 27,1 \\
\hline ID3 (80-89) & 497 & 35,2 \\
\hline ID4 (90 e mais) & 229 & 16,2 \\
\hline \multicolumn{3}{|l|}{ Sexo } \\
\hline FEM (Feminino) & 745 & 52,8 \\
\hline MAS (Masculino) & 666 & 47,2 \\
\hline \multicolumn{3}{|l|}{ Escolaridade (anos de estudo) } \\
\hline ES1 (1-3) & 425 & 30,1 \\
\hline ES2 (4-7) & 278 & 19,7 \\
\hline ES3 (8-11) & 118 & 8,4 \\
\hline ES4 (12 e mais) & 109 & 7,7 \\
\hline NEH (nenhuma escolaridade) & 301 & 21,3 \\
\hline ESG (escolaridade ignorada) & 180 & 12,8 \\
\hline \multicolumn{3}{|c|}{ Mês de ocorrência (estação sazonal) } \\
\hline CAL (calor) & 824 & 58,4 \\
\hline FRI (frio) & 587 & 41,6 \\
\hline \multicolumn{3}{|l|}{ Raça/Cor } \\
\hline BRA (branca) & 718 & 50,9 \\
\hline IMA (amarela/indígena) & 5 & 0,4 \\
\hline PAR (parda) & 423 & 30,0 \\
\hline PRE (preta) & 125 & 8,9 \\
\hline RAG (raça ignorada) & 140 & 9,9 \\
\hline \multicolumn{3}{|l|}{ Estado civil } \\
\hline CAS (casado) & 497 & 35,2 \\
\hline SOL (solteiro) & 283 & 20,1 \\
\hline VIU (viúvo) & 615 & 43,6 \\
\hline ECG (estado civil ignorado) & 16 & 1,1 \\
\hline
\end{tabular}

(continua) 
Tabela 1 (continuação)

\begin{tabular}{|c|c|c|}
\hline Variáveis/Categorias & Freqüência & $\%$ \\
\hline \multicolumn{3}{|l|}{ Local de ocorrência } \\
\hline DOM (domicílio) & 210 & 14,9 \\
\hline HOS (hospital) & 1.171 & 83,0 \\
\hline LOG (local ignorado) & 6 & 0,4 \\
\hline \multicolumn{3}{|l|}{ Distrito de residência } \\
\hline BAR (Barreiro) & 121 & 8,6 \\
\hline CSU (Centro-sul) & 181 & 12,8 \\
\hline LES (Leste) & 233 & 156 \\
\hline NDT (Nordeste) & 183 & 13,0 \\
\hline NOR (Norte) & 90 & 6,4 \\
\hline NRT (Noroeste) & 223 & 15,8 \\
\hline OET (Oeste) & 166 & 118 \\
\hline PAM (Pampulha) & 81 & 5,7 \\
\hline VNO (Venda Nova) & 133 & 9,4 \\
\hline \multicolumn{3}{|l|}{ Assistência médica } \\
\hline AMS (recebeu assistência médica) & 1.006 & 71,3 \\
\hline AMN (não recebeu assistência médica) & 6 & 0,6 \\
\hline AMG (assistência médica ignorada) & 399 & 28,3 \\
\hline \multicolumn{3}{|l|}{ Desnutrição } \\
\hline DPC (desnutrição protéico-calórica) & 1.384 & 98,1 \\
\hline MRT (marasmo nutricional) & 22 & 1,6 \\
\hline KWH (desnutrição protéica) & 5 & 0,4 \\
\hline
\end{tabular}

Nota: total de óbitos de idosos com desnutrição = 1.411 .

os meses frios. Esses dados foram fornecidos pela Seção de Observação e Meteorologia Aplicada do 5o Distrito de Meteorologia, Instituto Nacional de Meteorologia (INMET). Para raça/cor, cinco categorias foram estabelecidas: BRA (branca), IMA (amarela e indígena), PAR (parda), PRE (preta) e RAG (raça ignorada). O estado civil foi assim definido: CAS (casado e união consensual), SOL (solteiro), VIU (viúvo e separado judicialmente) e ECG (estado civil ignorado). A decisão pelo agrupamento das categorias união consensual e separado judicialmente às categorias casado e viúvo, respectivamente, foi decorrente da baixa freqüência apresentada pelas primeiras e pela maior similaridade em relação às últimas. Para o local de ocorrência do óbito, quatro categorias foram criadas: DOM (domicílio), HOS (hospital), OTR (outro local) e LOG (local ignorado). Os distritos de residência foram os definidos pela Organização Territorial dos Serviços de Saúde em Belo Horizonte 18, a saber: BAR (Barreiro), CSU (Centro Sul), LES (Leste), NDT (Nordeste), NOR (Norte), NRT (Noroeste), OET (Oeste), PAM (Pampulha) e VNO (Venda Nova). Por último, a variável assistência médica foi caracterizada em três categorias: AMS (recebeu assistência médica), AMN (não recebeu assistência médica) e AMG (assistência médica ignorada). Para estudo das associações, as categorias ignoradas de todas as variáveis foram eliminadas. A variável assistência médica foi excluída também do estudo de associações pelo fato de quase um terço das informações ser ignorado.

A causa básica de morte foi definida, conforme preconizado pela Organização Mundial da Saúde (OMS), como a doença ou lesão que iniciou a cadeia de eventos patológicos e que levou diretamente à morte 17 . As causas múltiplas foram definidas como o conjunto de todas as causas mencionadas na Declaração de Óbito, sem distinção de classificação, se básica, consequenciais ou contribuintes, conforme proposto por Laurenti 14 e Santo ${ }^{13}$. Para os diagnósticos mencionados na Declaração de Óbito, foi estabelecida a razão entre causa básica e causas múltiplas.

O método estatístico utilizado para estudo das associações foi o de análise de dados multidimensionais por meio da técnica de análise fatorial de correspondência múltipla 19 (Sampaio IBM. Relatório das atividades de pós-doutorado desenvolvidas no período de 03/set/92 a 04/ mar/93. Relatório de pós-doutorado apresentado ao Grupo de Estadística, Universidad Politécnica de Madrid). A técnica permite, por meio de combinações lineares, colocar em evidência os 
efeitos existentes nas modalidades combinadas, bem como as relações e associações entre as variáveis analisadas. Cada variável possui categorias distintas e exclusivas, de modo que cada observação (indivíduo) só pode pertencer a uma categoria única. Os resultados são expressos graficamente, sem testes inferenciais, em sistemas de eixos cartesianos. Cada eixo é uma combinação linear de todas as variáveis estudadas. A representação gráfica das variáveis exige o cálculo dos eixos fatoriais, das coordenadas destas sobre os referidos eixos e do percentual da inércia explicada. Os primeiros eixos fatoriais constituem o subespaço que melhor se ajusta à nuvem de pontos, ou seja, que menos a deforma quando ela se projeta sobre esse espaço. Se existirem "P" variáveis, o sistema consegue justificar-se plenamente com "P" eixos. Entretanto, para apreciação do fenômeno, utiliza-se uma dimensão perceptível (até três eixos), o que deve justificar uma variação das projeções acima de $70 \%$ da inércia total. A interpretação gráfica é baseada na localização das variáveis. Estão associadas aquelas encontradas na mesma região do espaço (quadrante) e na mesma direção em relação à origem. A distância entre elas será avaliada relativamente. Variáveis mais afastadas dos eixos contribuem mais para a variação. Variáveis próximas, em mesmo quadrante, usufruem das mesmas condições observadas e variáveis em quadrantes opostos serão antagônicas.

Para o processamento dos dados e análise estatística, utilizou-se o programa SAS (SAS Inst., Cary, Estados Unidos).

\section{Considerações éticas}

A base de dados do SIM foi disponibilizada pela Secretaria Municipal de Saúde de Belo Horizonte. Foram utilizados dados anônimos, somente para o objetivo proposto neste estudo, e as informações serão apresentadas de forma coletiva, com desagregação máxima por Distrito Sanitário.

\section{Resultados}

Foi registrado no período de 2000 a 2003 um total de 28.861 óbitos de pessoas idosas, com 60 anos e mais, residentes em Belo Horizonte. A desnutrição foi selecionada como causa básica e como causa múltipla em, respectivamente, 294 e 1.411 Declarações de Óbito (Tabela 2). Assim, o número de óbitos analisados neste estudo foi

Algumas causas múltiplas de morte mais freqüentes, causa básica e razão (causa múltipla/causa básica). Óbitos de idosos com desnutrição. Belo Horizonte, Minas Gerais, Brasil, 2000 a 2003.

\begin{tabular}{|c|c|c|c|}
\hline Causas de morte (CID-10) & Causa múltipla & Causa básica & Razão \\
\hline Desnutrição (E40-E46) & 1.411 & 294 & 4,80 \\
\hline Insuficiência respiratória (J96) & 446 & 15 & 29,73 \\
\hline Pneumonia (J12-J18) & 331 & 6 & 55,17 \\
\hline Septicemia (A40-A41) & 304 & 17 & 17,88 \\
\hline Doenças cerebrovasculares (160-169) & 293 & 182 & 1,61 \\
\hline úlcera de decúbito (L89) & 179 & 31 & 5,77 \\
\hline Pneumonia de aspiração (J69) & 137 & 24 & 5,71 \\
\hline Desidratação (E86) & 118 & 11 & 10,73 \\
\hline Doenças crônicas das vias aéreas inferiores (J40-J47) & 118 & 81 & 1,46 \\
\hline Insuficiência renal (N17-N19) & 105 & 19 & 5,53 \\
\hline Insuficiência cardíaca (I50) & 100 & 31 & 3,23 \\
\hline Doenças hipertensivas (I10-I13) & 86 & 14 & 6,14 \\
\hline Anemias (D50-D64) & 80 & 15 & 5,33 \\
\hline Diabetes mellitus (E10-E14) & 73 & 27 & 2,70 \\
\hline Infecção do trato urinário (N390) & 67 & 19 & 3,53 \\
\hline Neoplasia maligna de estômago (C16) & 46 & 42 & 1,09 \\
\hline Doença de Alzheimer (G30) & 45 & 30 & 1,50 \\
\hline Neoplasia de próstata (C61) & 44 & 36 & 1,22 \\
\hline Transtornos devido ao uso álcool (F10) & 42 & 15 & 2,80 \\
\hline Insuficiência hepática e outras doenças do fígado (K71-K73; K75-K76) & 37 & 6 & 6,17 \\
\hline
\end{tabular}

Nota: excluídas, na ordem, as causas de morte não especificadas e mal definidas. 
1.411, correspondente ao total de Declarações de Óbito de idosos em que a desnutrição foi mencionada, independente de sua posição ou classificação.

A desnutrição protéico-calórica representou 98\% dos casos de desnutrição. O número total de diagnósticos por Declaração de Óbito variou de 1 a 9, em média 4,1 por Declaração, totalizando 5.846 menções (causas múltiplas). Cerca de 70\% das Declarações de Óbito tinham quatro ou mais diagnósticos registrados e em apenas uma foi registrado um único diagnóstico. Os três diagnósticos que apresentaram maior razão entre causas múltiplas e causa básica foram pneumonia ( $R=55,17)$, insuficiência respiratória $(R=29,73)$ e septicemia $(\mathrm{R}=17,88)$. Outros diagnósticos que também apresentaram razão elevada foram desidratação $(R=10,73)$, doenças hipertensivas $(\mathrm{R}=6,14)$, úlcera de decúbito $(\mathrm{R}=5,77)$, pneumonia de aspiração $(R=5,71)$, anemias $(R=5,33)$ e desnutrição $(\mathrm{R}=4,8)$, conforme mostra a Tabela 2. A senilidade, apesar de não ter sido selecionada como causa básica, foi mencionada em 11,3\% das Declarações de Óbito.

A pesquisa mostrou sobremortalidade feminina $(52,8 \%)$, maior concentração de mortes na raça/cor branca $(50,9 \%)$, em viúvos $(43,6 \%)$ e na faixa etária de $80-89$ anos $(35,2 \%)$. Cerca de $21 \%$ dos idosos eram analfabetos e $30 \%$ tinham menos de três anos de estudo. O nível de escolaridade e a informação sobre assistência médica foram ignorados em 13\% e 28\% das Declarações de Óbito, respectivamente. A maior freqüência dos óbitos $(58,4 \%)$ ocorreu na estação quente e $15 \%$, no domicílio (Tabela 1 ).

Alguns perfis de mortalidade identificados são descritos a seguir.

Na Figura 1a, quatro perfis se destacaram. A inércia explicada foi de $20,1 \%$.

Idosos desnutridos do sexo masculino, com 4-7 anos de estudo, casados, morreram por desnutrição protéica na estação fria.

Mulheres idosas desnutridas, solteiras e viúvas, com 90 anos e mais e nenhuma escolaridade, morreram no calor, especialmente no Norte e Noroeste de Belo Horizonte.

Idosos desnutridos com alta escolaridade, cor branca, 80-89 anos morreram com desnutrição protéico-calórica, em domicílios concentrados nas regiões Centro-sul e Oeste.

Idosos desnutridos mais jovens (60-69 anos), das regiões Pampulha, Nordeste, Barreiro e Venda Nova, com baixa escolaridade, cor preta e parda, morreram com marasmo nutricional em hospitais.

Outros perfis de mortalidade foram identificados pelas inter-relações da desnutrição com neoplasias (Figura 1b) e com outras doenças (Fi- gura 1c). As inércias encontradas foram de 20,8\% e $19,8 \%$, respectivamente.

O marasmo nutricional mostrou-se associado a óbitos de idosos mais jovens, com neoplasias de boca, estômago, esôfago, reto, ânus, pulmão (Figura 1b); com diabetes mellitus, hipertensão arterial, insuficiência renal e com complicações da assistência médica e cirúrgica (Figura 1c);

Idosos mais velhos, com 80 anos e mais, morreram por desnutrição protéico-calórica, anemia, pneumonia, aterosclerose, fratura de fêmur, infecção do trato urinário e senilidade (Figura 1c).

Mortes de mulheres idosas desnutridas, com 90 anos e mais, foram associadas à neoplasia de mama (Figura 1b) e doenças cerebrovasculares, pneumonia de aspiração, úlcera de decúbito e imobilidade (Figura 1c).

Homens idosos desnutridos morreram mais freqüentemente por neoplasias de fígado, próstata e bexiga (Figura 1b) e por doenças isquêmicas do coração e das vias aéreas inferiores (Figura 1c).

As Figuras 1d e 1e (inércia de 18,2\% e 32,8\% respectivamente) mostram perfis relacionados a transtornos mentais pelo uso do álcool e fumo, além de doença de Alzheimer.

Idosos de 60-69 anos, com baixa escolaridade, cor preta e parda, das regiões Pampulha, Nordeste, Barreiro e Venda Nova, morreram em hospitais, no calor, por marasmo nutricional associado ao alcoolismo e tabagismo (Figura 1d).

A doença de Alzheimer foi mais bem evidenciada em idosos de 80-89 anos, cor branca, portadores de desnutrição protéica, que morreram no domicílio (Figura 1e).

\section{Discussão}

Os distúrbios nutricionais, especialmente a desnutrição protéico-calórica, tradicionalmente, têm sido mais enfocados na infância. Mais recentemente, o estudo do tema em pessoas idosas tem merecido maior atenção, considerando o aumento da expectativa de vida e os desafios colocados pelo envelhecimento. A análise do estado nutricional de idosos torna-se mais complexa em virtude da maior heterogeneidade deste grupo, seja pelas modificações biológicas da idade, pela influência de fatores sócio-econômicos, seja por hábitos praticados ao longo da vida, como bebida, fumo, dieta e atividades físicas. Correia \& Waitzberg 20 evidenciaram que as complicações em desnutridos é 1,6 vez maior que em eutróficos e que a mortalidade nos primeiros é 2,6 vezes maior.

Quantificar a magnitude da desnutrição entre idosos não tem sido tarefa fácil pelas dificuldades inerentes aos estudos de morbidade e pelo fato 
de as estatísticas oficiais de mortalidade serem baseadas em apenas uma causa - a causa básica de morte -, ocasionando uma grande perda de informações. Ao se propor, neste trabalho, analisar a desnutrição sob o enfoque de causas múltiplas de morte, pretendeu-se captá-la, independentemente da posição ocupada na Declaração de Óbito: se mencionada como causa básica, conseqüencial ou contribuinte. As afecções originadas a partir da causa básica, na Parte I do Atestado Médico, são denominadas causas consequenciais, das quais a última mencionada é a causa terminal ou imediata. Na Parte II, são descritas as causas contribuintes, que não entram na cadeia de eventos iniciada pela causa básica, mas que, juntamente com as causas consequenciais, constituem as causas associadas. As causas múltiplas representam a união da causa básica com as causas associadas 13 .

Neste estudo, se a desnutrição fosse analisada sob o enfoque da causa básica, os óbitos com menção dessa causa seriam subdimensionados cerca de quase cinco vezes, o que reforça a sua importância como causa contribuinte ou associada de morte, conforme já evidenciado em outros estudos 13,21,22. Santo 22 acrescenta que a desnutrição aparece como causa básica em menos de $40 \%$ das vezes em que é mencionada na Declaração de Óbito. Por esse motivo, Otero et al. 8, em 2002, sugeriram o aprofundamento do estudo da desnutrição como causa de morte em idosos, de forma preferencial sob o enfoque de causas múltiplas, para se conhecer a real magnitude desta causa. Entretanto, em qualquer dos dois enfoques para análise de causas de morte, as estatísticas de mortalidade só terão validade se a Declaração de Óbito for preenchida de forma correta. A qualidade da informação, em grande parte, depende da importância dada pelo médico à Declaração de Óbito como fonte de dados de mortalidade. Há de se ressaltarem, ainda, as limitações de natureza quantitativa, na qual o sub-registro é o fator mais importante da baixa cobertura 22,23.

$\mathrm{O}$ alto percentual de Declarações de Óbito com quatro ou mais diagnósticos demonstra, por um lado, o acúmulo de doenças nos idosos e, por assim ser, a grande perda de informações quando as análises de mortalidade, especialmente para esse estrato da população, são feitas sob a perspectiva de causa básica. A média de 4,1 diagnósticos por declaração superou em muito a média de 2,6 encontrada por Rezende et al. 24 para a população de 20 anos e mais, também de Belo Horizonte. Neste caso, o percentual de declarações com quatro ou mais diagnósticos não chegou a $20 \%$, contrastando com os $70 \%$ registrados no presente estudo, o que, por outro lado, pode expressar igualmente a qualidade do preenchimento do atestado médico.

Além da desnutrição, as maiores razões registradas entre causas múltiplas e causa básica para pneumonias, insuficiência respiratória, septicemias, desidratação, doenças hipertensivas, úlcera de decúbito e pneumonia de aspiração demonstram que essas causas de morte foram mais bem captadas pelo enfoque de causas múltiplas. Sabe-se que a estrutura e a função do parênquima pulmonar podem ser alteradas pela desnutrição, deixando o tecido pulmonar mais susceptível à lesão ${ }^{25}$. Isto pode justificar o fato de os problemas respiratórios serem os mais freqüentemente mencionados. Além disso, a pneumonia, insuficiência respiratória e septicemia podem ser devidas ao fato de serem causas terminais ou complicações comuns de outras patologias. A desnutrição protéico-calórica pode alterar também a quantidade e composição de fluidos teciduais, resultando em desidratação, choque e morte 11. A senilidade, por englobar as causas mal definidas, somente foi captada sob o enfoque de causas múltiplas, já que, pelas regras da CID10, não pode ser selecionada como causa básica de morte, a não ser quando aparece como única causa mencionada na Declaração de Óbito 26. Ao figurar entre as dez primeiras causas múltiplas de morte suscita a necessidade de melhor especificação e descrição da causa específica da morte 27. Para Santo 13, senilidade e anemias são causas contribuintes, com baixa letalidade, que aparecem coligadas a outras doenças; septicemia e desidratação são causas consequenciais determinadas por outras afecções; e a hipertensão arterial, preterida nas regras de seleção de causa básica pela CID-10, é bem dimensionada nos estudos de causas múltiplas.

A sobremortalidade feminina, possivelmente pela maior incidência de mulheres idosas, e a baixa escolaridade registrada caracterizam o perfil dos idosos desnutridos também descrito em outros estudos 28. A maior mortalidade feminina, raça branca, faixa etária de 80-89 anos e viúvos podem estar refletindo o perfil demográfico da população idosa de Belo Horizonte. Contudo, taxas de mortalidade por desnutrição em homens idosos mostraram-se mais elevadas, quando comparadas às das mulheres 29 . O analfabetismo e a pouca escolaridade podem retratar dificuldades na formulação de conceitos de saúde importantes para o autocuidado. A maior concentração de mortes em viúvos não permite fazer inferências em relação ao autocuidado e à autoestima, já que não foi objeto de estudo a informação sobre viver sozinho ou não. No entanto, não se pode descartar a possibilidade de que o isolamento e o abandono do idoso tenham 
Figura 1

Representação gráfica das variáveis estudadas nos dois primeiros eixos da análise fatorial de correspondência, no universo de mortes de idosos com desnutrição. Belo Horizonte, Minas Gerais, Brasil, 2000 a 2003.

1a)

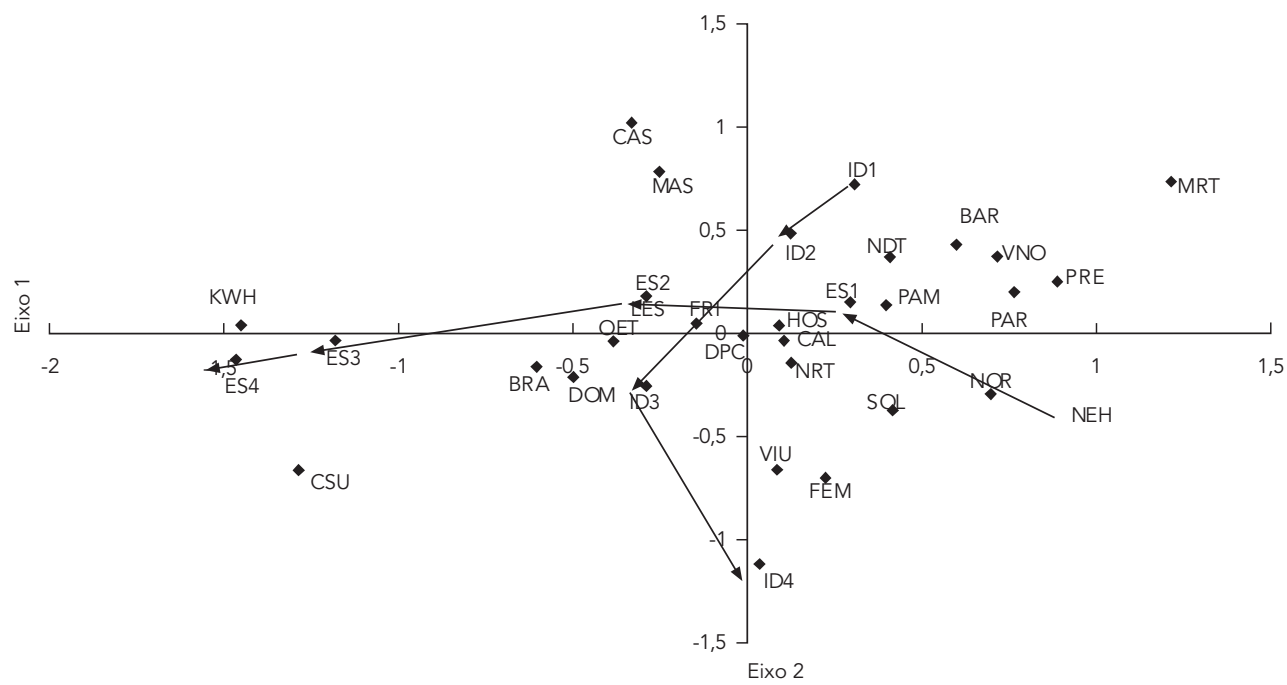

1b)

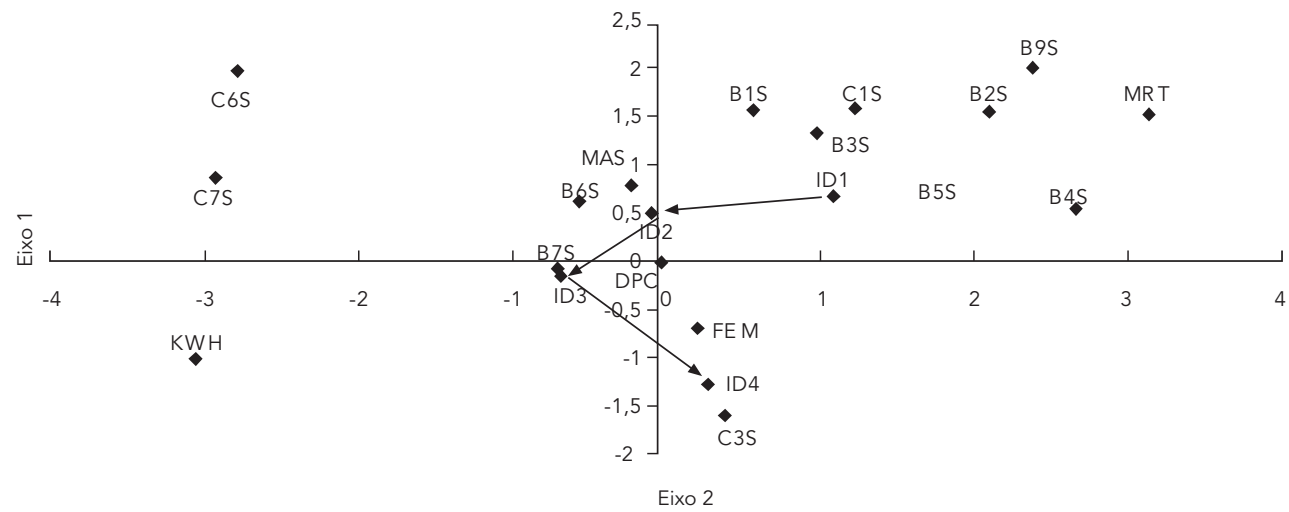

1c)

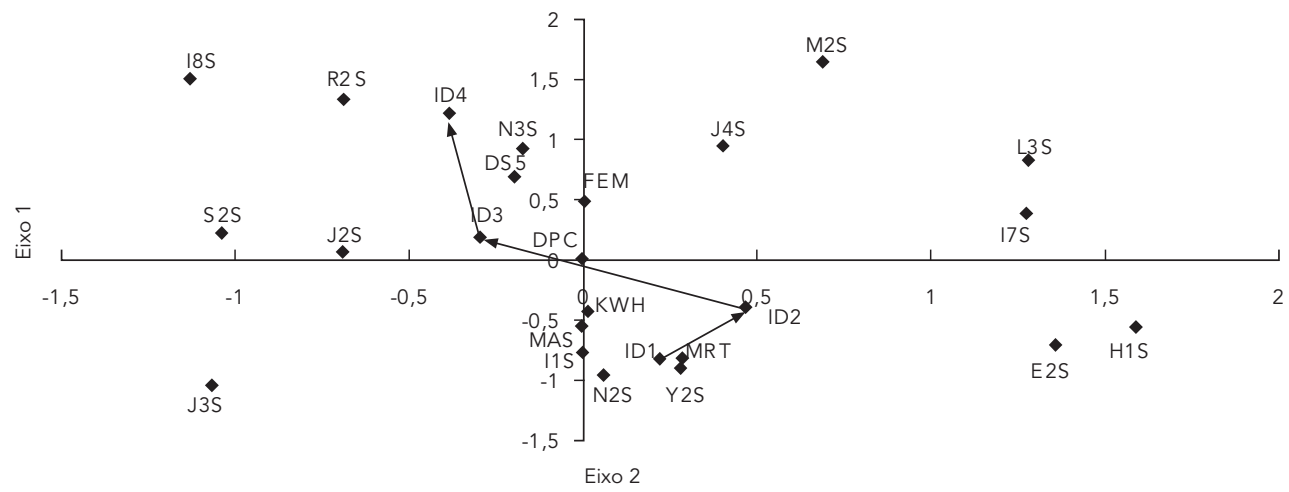

(continua) 


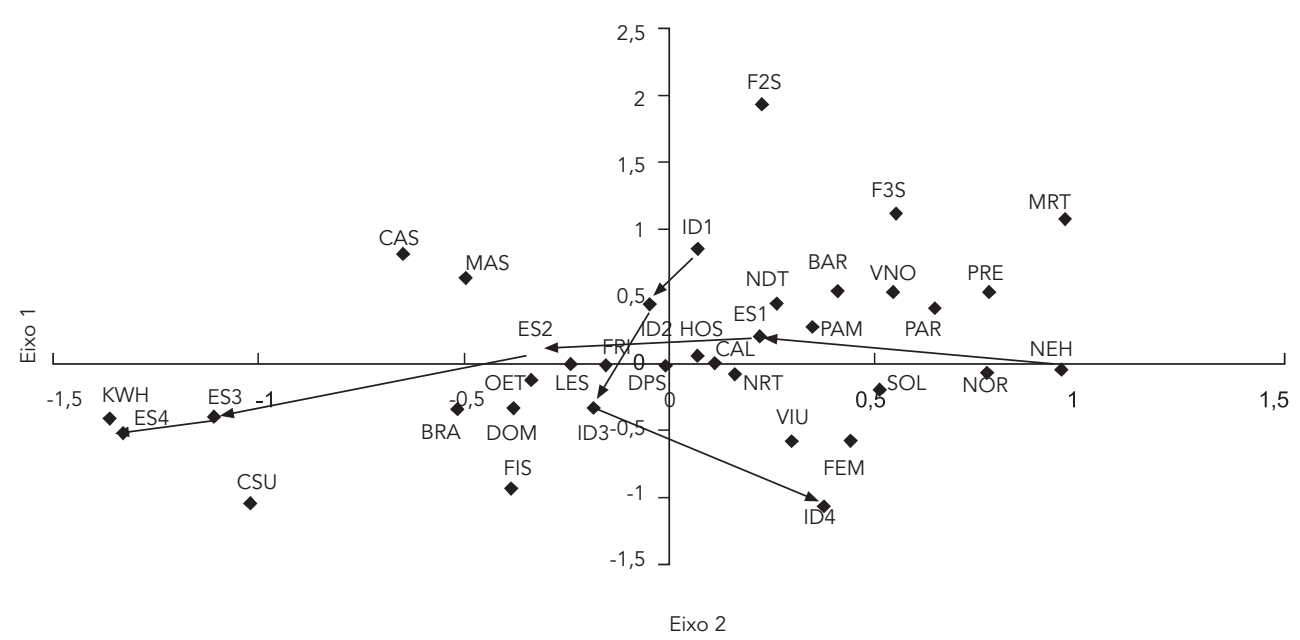

1e)

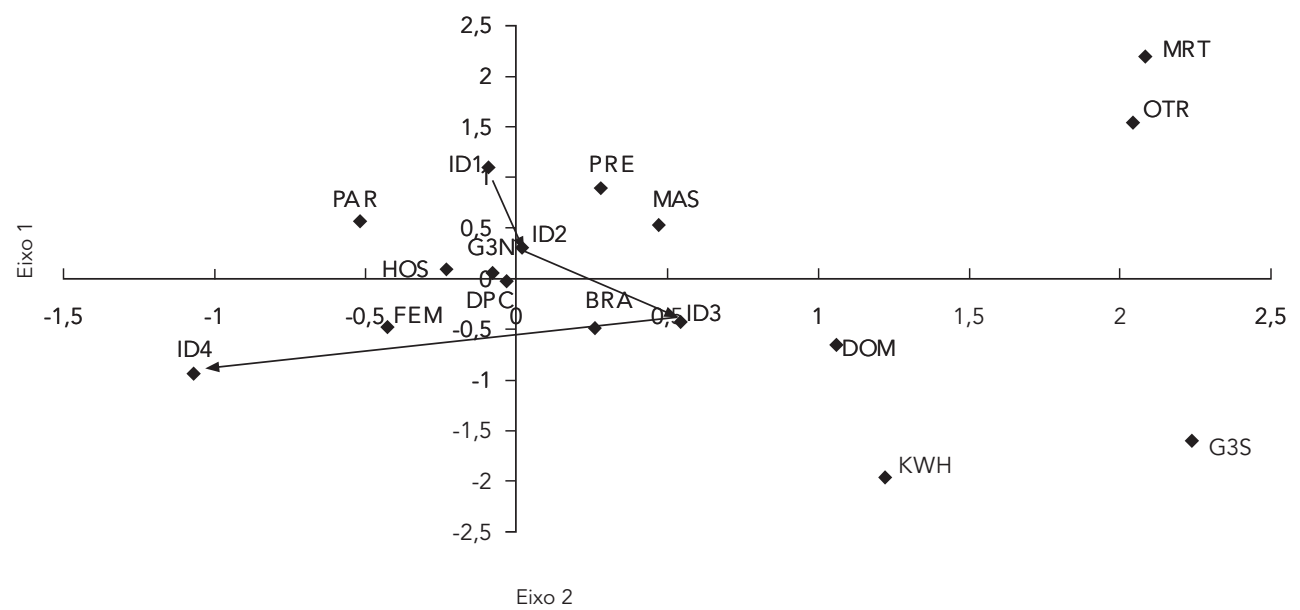

B1S: neoplasia de cavidade oral e faringe; B2S: neoplasia de esôfago; B3S: neoplasia de estômago; B4S: outras neoplasias digestivas; B5S: neoplasia de cólon, reto e ânus; B6S: neoplasia de fígado e vias biliares; B7S: neoplasia de pâncreas;

B9S: neoplasias de laringe; BAR: Barreiro; BRA: branca; C1S: neoplasia de traquéia, brônquios e pulmões; C3S: neoplasia de mama; C6S: neoplasia de próstata; C7S: neoplasia de bexiga; CAL: calor; CAS: casado; CSU: Centro-sul; D5S: anemias; DOM: domicílio; DPC: desnutrição protéico-calórica; E2S: diabetes mellitus; ES1: 1-3 anos de estudo; ES2: 4-7 anos de estudo; ES3: 8-11 anos de estudo; ES4: 12 e mais anos de estudo; F1S: restante dos transtornos mentais e comportamentais; F2S: transtornos mentais devido ao uso de álcool; F3S: transtornos mentais devido ao uso do fumo; FEM: feminino; FRI: frio; G3S: doença de Alzheimer; H1S: doenças hipertensivas; HOS: hospital; I1S: doenças isquêmicas do coração; 17S: doenças cerebrovasculares; I8S: aterosclerose; ID1: 60-69 anos; ID2: 70-79 anos; ID3: 80-89 anos; ID4: 90 anos e mais; J2S: pneumonia; J3S: doenças crônicas das vias aéreas inferiores; J4S: pneumonia de aspiração; KWH: desnutrição protéica; L3S: úlcera de decúbito; LES: Leste; M2S: síndrome de imobilidade; MAS: masculino; MRT: marasmo nutricional; N2S: insuficiência renal; N3S: infecção do trato urinário; NDT: Nordeste; NEH: nenhuma escolaridade; NOR: Norte; NRT: Noroeste; OET: Oeste; OTR: outro local; PAM: Pampulha; PAR: parda; PRE: preta; R2S: senilidade; S2S: fratura de fêmur; SOL: solteiro; VIU: viúvo/separado; VNO: Venda Nova; Y2S: complicações de assistência médica e cirúrgica. 
contribuído para o óbito, uma vez que estudos mostram que a desnutrição pode ser decorrente da pobreza, da depressão, de morar sozinho e de isolamento social, o que pode ocorrer com mais freqüência entre viúvos 10 .

$\mathrm{O}$ alto percentual de idosos nas faixas etárias acima de 80 anos demonstra a capacidade de sobrevida, mesmo com o acometimento simultâneo de várias patologias, aspecto também observado nos estudos de Otero et al. ${ }^{8}$ e Otero 21 , ao demonstrarem que $77 \%$ dos óbitos ocorriam em idosos acima de 70 anos.

A maior freqüência de óbitos na estação quente é compatível com os resultados encontrados por Otero et al. 15 para a cidade do Rio de Janeiro, mas contrária aos seus achados para a Região Metropolitana de São Paulo. Segundo o autor, os extremos de temperatura constituem um estresse adicional para os idosos desnutridos. Melhor influência dessa variável será evidenciada nas associações com outros fatores e patologias. Como a informação sobre a assistência médica recebida foi ignorada em cerca de um terço das Declarações de Óbito, não se pode estudar as associações envolvendo a participação dessa variável.

Uma das dificuldades no estudo de associações de causas de morte, como ressaltam Olson et al. 30, tem sido determinar a significância estatística, visto que os métodos utilizados partem do pressuposto de independência das variáveis, portanto não aplicáveis ao enfoque de causas múltiplas, cuja premissa é a interação entre as causas. Por essa razão, o método de análise de dados multidimensionais sugerido por Judez $19 \mathrm{e}$ Sampaio (Sampaio IBM. Relatório das atividades de pós-doutorado desenvolvidas no período de 03/set/92 a 04/mar/93. Relatório de pós-doutorado apresentado ao Grupo de Estadística, Universidad Politécnica de Madrid), por não utilizar testes inferenciais, foi o escolhido para este estudo. Foi utilizado por Rezende et al. ${ }^{24}$ para análise de associações de causas múltiplas de morte por doenças crônico-degenerativas e recomendado para análises similares.

Optou-se pela técnica de análise de correspondência múltipla para estabelecer padrões de morbimortalidade, utilizando as causas múltiplas de morte, o que permitiu observar, com mais propriedade, os conjuntos de enfermidades que determinaram o óbito. A técnica usada permitiu captar, especialmente pelas representações gráficas, as relações entre essas enfermidades e ainda as suas inter-relações com outras variáveis. O fato de o total da inércia nos três primeiros eixos fatoriais ter ficado abaixo de $70 \%$, não compromete as inter-relações encontradas, já que, mesmo com justificativas baixas da inér- cia, possivelmente pela instabilidade da resposta medida, as associações buscadas podem claramente se manifestar (Sampaio IBM. Relatório das atividades de pós-doutorado desenvolvidas no período de 03/set/92 a 04/mar/93. Relatório de pós-doutorado apresentado ao Grupo de Estadística, Universidad Politécnica de Madrid). O que se observa é que, em estudos de associações de causas de morte, o percentual de explicação da inércia para projeção das variáveis nos três primeiros eixos fatoriais é baixo, como evidenciado em estudos similares 16,31.

Dois perfis de mortalidade bem demarcados neste estudo é o de idosos desnutridos com alta escolaridade, cor branca, 80-89 anos, que morreram por desnutrição protéico-calórica, em domicílios, e o de idosos desnutridos mais jovens (60-69 anos), com baixa escolaridade, cor preta e parda, que morreram por marasmo nutricional, em hospitais. No primeiro caso, os óbitos foram concentrados nas regiões Centro-sul e Oeste de Belo Horizonte, regionais com maior concentração de idosos em melhor situação sócio-econômica ${ }^{32}$, compatível, portanto, com as características descritas. No segundo caso, os idosos eram residentes dos distritos Pampulha, Nordeste, Barreiro e Venda Nova, regiões que, com exceção da Pampulha, apresentavam pior situação sócioeconômica 32 , o que pode ter contribuído para a precocidade das mortes. A associação de óbitos desses idosos mais jovens com diabetes mellitus, hipertensão arterial e insuficiência renal sugere um pior acesso a serviços de saúde.

Estudo para avaliar a carência social em idosos em Recife, Pernambuco, revelou que idosos com desnutrição protéico-calórica apresentam risco crescente com o aumento da carência social. Aqueles que residiam em áreas de muito alta carência social apresentaram risco 9,6 vezes maior de morrer por desnutrição do que os residentes em bairros de baixa carência social 4 . A desnutrição protéica, acometendo mais freqüentemente idosos mais velhos (80 anos e mais), também se associou a pneumonia, aterosclerose, fratura de fêmur, infecção do trato urinário, senilidade e doença de Alzheimer. Louvison et al. 33 , ao estudarem os fatores relacionados à determinação e às desigualdades no acesso e uso dos serviços de saúde por idosos no Município de São Paulo, constataram que o melhor nível sócioeconômico favorece maior acesso a serviços de saúde e conseqüentes diagnóstico e tratamento precoces. Isto, por sua vez, refletiria na adoção de comportamentos que levam a melhor situação de saúde e, conseqüentemente, a maior expectativa de vida. Estes achados confirmam a importância do investimento nas ações de saúde, dentre elas, as ações do Programa Saúde da Família 34 . 
Por sua vez, o marasmo nutricional em idosos mais jovens mostrou-se mais associado às neoplasias, especialmente às do sistema digestivo (esôfago e estômago), o que limita a ingestão e absorção dos alimentos, ocasionando perda rápida de peso. Foi também evidente neste grupo a associação dos óbitos com o alcoolismo e o tabagismo, já demonstrada em outros estudos que enfocaram as causas múltiplas de morte 13,16. A associação de óbitos com o tabagismo foi estatisticamente significativa em estudo de correlação de avaliação nutricional e complicações pós-operatórias em neoplasias de cabeça e pescoço ${ }^{35}$.

Há ainda dois outros perfis caracterizados que se contrapõem em relação ao sexo e ao período de ocorrência do óbito: idosos desnutridos do sexo masculino, casados, que morreram por desnutrição protéica na estação fria, e mulheres idosas, com 90 anos e mais, desnutridas, viúvas, que morreram no calor. Otero 21, ao estudar séries históricas de óbitos por desnutrição em São Paulo e Rio de Janeiro, detectou a existência de um padrão sazonal no inverno, para São Paulo, e no verão, para o Rio de Janeiro. Ressaltou que idosos desnutridos podem ter dificuldade de manter a temperatura do corpo normal nos meses frios, já que o baixo peso compromete a proteção térmica do organismo. Assim, o idoso estaria mais suscetível a diversos agravos, especialmente aqueles de origem respiratória. Os efeitos do calor, por outro lado, podem se manifestar sobre as doenças circulatórias 36 , as quais são imediatas sobre a mortalidade 37 . Os estudos citados não mostraram, entretanto, influências sazonais em relação ao sexo, como evidenciado neste estudo. Ainda referente ao sexo, observou-se que mulheres idosas desnutridas morreram por neoplasia de mama, doenças cerebrovasculares, pneumonia de aspiração, úlcera de decúbito e imobilidade. A morte de homens idosos desnutridos mostrouse associada a neoplasias do fígado, próstata e bexiga, além de doenças isquêmicas do coração e das vias aéreas inferiores. Essas associações com a desnutrição são passíveis de explicação pela doença de base e ainda pela maior freqüência ocorrida em cada sexo.

\section{Conclusão}

Foi possível verificar neste estudo, pelo número médio de diagnósticos referidos por Declaração de Óbito, que os idosos morreram com uma variedade de causas, dificultando a escolha de apenas uma para determinar a morte. Demonstrou-se, dessa forma, a limitação da análise que enfoca apenas a causa básica, tradicionalmente utilizada nas estatísticas oficiais de mortalidade, e o impacto da abordagem de causas múltiplas, ao evidenciar causas de morte que permaneceriam ocultas ou subdimensionadas pelo enfoque tradicional. A análise multidimensional permitiu estudar as associações de causas de morte e, a partir daí, identificar perfis de mortalidade.

O melhor entendimento do conjunto de enfermidades que desencadeou o óbito de idosos com desnutrição poderá subsidiar medidas de intervenção direcionadas à assistência, prevenção e promoção de saúde. Os perfis de mortalidade identificados devem ser repensados, na prática, por instituições e profissionais de saúde, a fim de redirecionar e priorizar ações de melhoria da qualidade de vida e, ainda, planejar e definir políticas públicas direcionadas ao bem-estar da população idosa. Outra sinalização importante deste estudo é a necessidade de melhoria do preenchimento da Declaração de Óbito, pois a análise de muitas variáveis ficou prejudicada pelo alto índice informações ignoradas. 


\section{Resumo}

O objetivo deste estudo foi identificar perfis de mortalidade em idosos com desnutrição, a partir de associações entre causas múltiplas de morte e outras variáveis da Declaração de Óbito. Foram estudadas as mortes de residentes de Belo Horizonte, Minas Gerais, Brasil, com 60 anos e mais, que tinham a desnutrição como uma das causas mencionadas. Utilizaram-se os dados do Sistema de Informações sobre Mortalidade. Para estudo das associações, foi empregada a análise multidimensional pela técnica de análise fatorial de correspondência múltipla. A pesquisa mostrou sobremortalidade feminina, maior concentração de mortes na raça branca, em viúvos e em idosos com baixa escolaridade. Foram estabelecidos dez perfis de mortalidade e a desnutrição foi evidenciada cerca de cinco vezes mais pelo enfoque das causas múltiplas. Confirmou-se a importância das causas múltiplas para evidenciar causas de morte não detectadas pelo enfoque de causa básica e a adequação da técnica de análise de correspondência múltipla para identificar as associações de causas e demais variáveis da Declaração de Óbito.

Idoso; Desnutrição; Causas Múltiplas de Morte

\section{Colaboradores}

E. M. Rezende participou da concepção da pesquisa análise e interpretação dos dados, redação e aprovação da versão final. I. B. M. Sampaio colaborou na concepção da pesquisa, no delineamento estatístico, na análise e interpretação dos dados. L. H. Ishitani contribuiu na análise e interpretação dos dados, redação, revisão crítica e aprovação da versão final. E. F. Martins e L. C. M. Vilella participaram da análise e interpretação dos dados, revisão crítica e aprovação da versão final.

\section{Agradecimentos}

Agradecemos à Seção de Observação e Meteorologia Aplicada do 5o Distrito de Meteorologia, Instituto Nacional de Meteorologia (INMET) de Belo Horizonte, pelo fornecimento dos dados referentes às temperaturas registradas no município, e à Pró-Reitoria de Pesquisa da Universidade Federal de Minas Gerais (UFMG), pelo financiamento.

\section{Referências}

1. Carvalho JAM, Garcia RA. O envelhecimento da população brasileira: um enfoque demográfico. Cad Saúde Pública 2003; 19:725-33.

2. Lima-Costa MF, Veras R. Saúde pública e envelhecimento. Cad Saúde Pública 2003; 19:700-1.

3. Rede Interagencial de Informação em Saúde. Indicadores básicos para a saúde no Brasil: conceitos e aplicações. 2a Ed. Brasília: Organização Pan-Americana da Saúde; 2008.

4. Silva VL, Leal MCC, Marino JG, Marques APO. Associação entre carência social e causas de morte entre idosos residentes no Município de Recife, Pernambuco, Brasil. Cad Saúde Pública 2008; 24:1013-23.

5. Karsch UM. Idosos dependentes: famílias e cuidadores. Cad Saúde Pública 2003; 19:861-6.

6. Czaka-Narins DM. Avaliação do estado nutricional. In: Mahan LK, Arlin MT, organizadores. Krause: alimentos, nutrição e dietoterapia. 8a Ed. São Paulo: Editora Roca; 1995. p. 309-30.
7. Alves DC. Desnutrição. In: Augusto ALP, Alves DC, Mannarino IC, Gerude M, organizadores. Terapia nutricional. São Paulo: Editora Atheneu; 2005. p. 220-9.

8. Fiore EG, Vieira VL, Cervato AM, Tucilo DR, Cordeiro AA. Perfil nutricional de idosos freqüentadores de unidade básica de saúde. Rev Ciênc Méd (Campinas) 2006; 15:369-77.

9. Sullivan DH, Sung S, Walls RC. Protein-energy undernutrition among elderly hospitalized patients. JAMA 1999; 281:2013-9.

10. Otero UB, Rosenfeld S, Gadelha AJ, Carvalho NS. Mortalidade por desnutrição em idosos, Região Sudeste do Brasil, 1980-1997. Rev Saúde Pública 2002; 36:141-8.

11. Marchini JS, Ferriolli E, Moriguti JC. Suporte nutricional no paciente idoso: definição, diagnóstico, avaliação e intervenção. Medicina (Ribeirão Preto) 1998; 31:54-61. 
12. Moriguti JC, Moriguti EKU, Ferriolli E, Cação JC, Iucif Junior N, Marchini JS. Involuntary weight loss in elderly individuals: assessment and treatment. São Paulo Med J 2001; 119:72-7.

13. Santo AH. Causas múltiplas de morte: formas de apresentação e métodos de análise [Tese de Doutorado]. São Paulo: Universidade de São Paulo; 1988.

14. Laurenti R. Causas múltiplas de morte [Tese de Livre Docência]. São Paulo: Faculdade de Saúde Pública, Universidade de São Paulo; 1973.

15. Otero UB, Rosenfeld S, Gadelha AJ. Óbitos por desnutrição em idosos em São Paulo e Rio de Janeiro: análise de séries temporais, 1980-1986. Rev Bras Epidemiol 2001; 4:1911-205.

16. Rezende EM. Análise multidimensional de causas múltiplas de óbitos humanos em Belo Horizonte 1998 [Tese de Doutorado]. Belo Horizonte: Universidade Federal de Minas Gerais; 2003.

17. Organização Mundial da Saúde. Classificação estatística internacional de doenças e problemas relacionados à saúde, 10ạ revisão. São Paulo: Centro Colaborador da OMS para a Classificação de Doenças em Português; 1995.

18. Secretaria Municipal de Saúde de Belo Horizonte. Organização territorial dos serviços de saúde em BeloHorizonte.http://www.pbh.gov.br/smsa/biblio teca/geepi/territoriosnasmsa.doc (acessado em 14/ Jan/2010).

19. Judez AL. Análisis factorial de correspondencias. In: Judez AL, editor. Técnicas de análisis de datos multidimensionales. Madrid: Ministério de Agricultura, Pesca y Alimentación; 1989. p. 69-114.

20. Correia MI, Waitzberg DL. The impact of malnutrition on morbidity, mortality, length of hospital stay and costs evaluated through a multivariate model analysis. Clin Nutr 2003 22:235-9.

21. Otero UB. Estudo da mortalidade por desnutrição em idosos na Região Sudeste do Brasil: 1980-1997 [Dissertação de Mestrado]. Rio de Janeiro: Universidade do Estado do Rio de Janeiro; 2001.

22. Santo AH. Potencial epidemiológico da utilização das causas múltiplas de morte por meio de suas menções nas declarações de óbito, Brasil, 2003. Rev Panam Salud Pública 2007; 22:178-86.

23. Laurenti R, Buchalla CM. A elaboração de estatísticas de mortalidade. Rev Bras Epidemiol 2000; 3: 21-8.

24. Rezende EM, Sampaio IBM, Ishitani LH. Causas múltiplas de morte por doenças crônico-degenerativas: uma análise multidimensional. Cad Saúde Pública 2004; 20:1223-31.

25. Adams E. Cuidado nutricional na doença pulmonar. In: Mahan LK, Arlin MT, organizadores. Krause: alimentos, nutrição e dietoterapia. 8a Ed. São Paulo: Editora Roca; 1995. p. 615-27.

26. Mello-Jorge MHP, Laurenti R, Lima-Costa MF, Gotlieb SLD, Chiavegatto Filho ADP. A mortalidade de idosos no Brasil: a questão das causas mal definidas. Epidemiol Serv Saúde 2008; 17:271-81.
27. Mathias TAF, Melo-Jorge MHP, Larenti R, Aidar T. Considerações sobre a qualidade de informações de mortalidade na população idosa residente no município de Maringá, Estado do Paraná, Brasil, no período de 1979 a 1998. Epidemiol Serv Saúde 2005; 14:159-69.

28. Pereira RS. Risco de desnutrição em idosos institucionalizados no município do Rio de Janeiro: um estudo com a Mini Avaliação Nutricional (MAN) [Dissertação de Mestrado]. Rio de Janeiro: Universidade do Estado do Rio de Janeiro; 2004.

29. Lima e Costa MF, Guerra HL, Barreto SM, Guimarães RM. Diagnóstico da situação de saúde da população idosa brasileira: um estudo da mortalidade e das internações hospitalares públicas. Inf Epidemiol SUS 2000; 9:23-41.

30. Olson FE, Norris FD, Hammes LM, Shipley PW. A study of multiple causes of death in California. J Chronic Dis 1962; 15:157-70.

31. Machado CJ. Mortalidade infantil no Estado de São Paulo, 1999: uma análise das causas múltiplas de morte a partir de componentes principais. Rev Bras Epidemiol 2004; 7:11-21.

32. Zahreddine D, Rigotti JIR. O processo de envelhecimento populacional em Belo Horizonte: análise e mapeamento dos anos de 1991 e 2000. In: Anais do XVEncontrodeEstudosPopulacionais.http://www. abep.nepo.unicamp.br/encontro2006/docspdf/ ABEP2006_787.pdf (acessado em 14/Jan/2010).

33. Louvison MCP, Lebrão ML, Duarte YAO, Santos JLF, Malik AM, Almeida ES. Desigualdades no uso e acesso aos serviços de saúde entre idosos do município de São Paulo. Rev Saúde Pública 2008; 42:733-40.

34. Silvestre JA, Costa Neto MM. Abordagem do idoso em programas de saúde da família. Cad Saúde Pública 2003; 19:839-47.

35. Oliveira BV, Ramos G, Dias LAN, Rossoni MD. Avaliação nutricional e complicações pós-operatórias em pacientes portadores de carcinomas de cabeça e pescoço. Acta Oncol Bras 1994; 14:159-66.

36. Lage Ferrón MB, Díaz Jiménez J, Gestal Otero JJ, de la Sierra Pajares Ortíz M, Alberdi Odriozola JC. Influencia de los factores ambientales en el número de ingresos por urgencias en el complejo hospitalario “Juan Canalejo" de La Coruña: elaboración de un modelo de predicción. Rev Esp Salud Pública 1999; 73:45-60.

37. Sierra Pajares Ortíz M, Díaz Jiménez J, Montero Rubio JC, Alberdi Odriozola JC, Mirón Pérez IJ. Mortalidad diaria en la Comunidad de Madrid durante el período 1986-1991 para el grupo de edad de 45 a 64 años: su relación con la temperatura del aire. Rev Esp Salud Pública 1997; 71:149-60.

Recebido em 24/Ago/2009

Versão final reapresentada em 15/Jan/2010

Aprovado em 01/Abr/2010 